\title{
O ensino da metodologia de investigação na formação inicial em Serviço Social: Entre a transversalidade exigida e a predominância da metodologia qualitativa
}

\author{
Daniela Monteiro' e Francisco Branco ${ }^{2}$ \\ ${ }^{1}$ Faculdade de Filosofia e Ciências Sociais Universidade Católica Portuguesa, Portugal | \\ dmonteiro@ucp.pt | https://orcid.org/0000-0002-3968-1531 \\ ${ }^{2}$ Faculdade de Ciências Humanas Universidade Católica Portuguesa, Portugal | \\ fnbranco@ucp.pt | https://orcid.org/0000-0003-0075-792X
}

\begin{abstract}
Resumo: O serviço social é uma profissão reconhecida pela sua intervenção junto das populações em situação de vulnerabilidade social. Mas, não se reduz a esta dimensão e a investigação tem ganho cada vez mais espaço na prática dos profissionais e destaque na literatura da área. Assim, importa compreender como formamos os estudantes e futuros profissionais neste domínio. Neste artigo, apresentamos uma parte de um estudo mais amplo sobre o ensino da investigação na formação inicial em serviço social, onde pretendemos conhecer e compreender as orientações metodológicas de investigação lecionadas na formação inicial em serviço social a partir da visão dos professores. Trata-se de um estudo de casos múltiplos de natureza qualitativa, cujas unidades de análise são três cursos de Licenciatura em Serviço Social em Portugal, onde foram entrevistados 12 professores. Os dados revelaram a preocupação que as IES (Instituições de Ensino Superior) demonstraram em garantir aos estudantes um conhecimento transversal das diferentes abordagens metodológicas, independentemente das tendências que marcam atualmente as investigações produzidas pelos assistentes sociais, o seu interesse ou adequação à área disciplinar e às metodologias preferencialmente adotadas pelos próprios professores enquanto investigadores. Conclui-se, ainda, que, não obstante esta transversalidade, verifica-se uma predominância da metodologia qualitativa nos trabalhos desenvolvidos pelos estudantes de serviço social numa associação com a natureza da intervenção da profissão.
\end{abstract}

Palavras-chave: Serviço Social; Formação Graduada; Metodologia de Investigação; Metodologia Qualitativa.

Teaching Research Methods in Initial Training in Social Work: between the Required Transversality and the Qualitative Methodology Predominance

Abstract: Social work is a profession recognized for its intervention with populations in situations of social vulnerability. However, it is not reduced to this dimension and research has gained more and more space in the practice of professionals and it is highlighted in the literature of the area. Thus, it is important to understand how we train students and future professionals in this field. In this article, we present a part of a broader study on the research teaching in initial training in social work, where we intend to know and understand the methodological guidelines of research taught in initial training in social work from the perspective of teachers. This is a multiple case study of a qualitative nature, whose units of analysis are three Degree courses in Social Work in Portugal, where 12 teachers were interviewed. The data revealed the concern that HEls (Higher Education Institutions) demonstrated in guaranteeing students a transversal knowledge of the different methodological approaches, regardless of the current tendencies that mark the investigations produced by social workers, their interest or suitability to the disciplinary area and the methodologies preferably adopted by the teachers themselves as researchers. It is also concluded that, despite this transversality, there is a predominance of qualitative methodology in the works developed by social work students in association with the nature of the profession's intervention.

Keywords: Social Work; Undergraduate Training; Research Methodology; Qualitative Methodology. 


\section{Introdução}

O serviço social é uma profissão de intervenção social que se vem afirmando como disciplina académica, onde o domínio da investigação ganha cada vez mais relevo, nomeadamente para a prática profissional. Contudo, a literatura aponta para uma falta de confiança dos assistentes sociais na realização de investigações, o que contribuiu para a baixa produção científica da área (Harvey et al., 2013). O mesmo pode ser verificado nos estudantes de serviço social nos diferentes ciclos do ensino superior (Adam et al., 2004; Maschi et al., 2013) que além da falta de confiança apresentam ansiedade em relação às unidades curriculares de Métodos e Técnicas de Investigação (Bolin et al., 2012; Einbinder, 2014; Maschi et al., 2013) e uma certa relutância relativamente a estas (D'Cruz \& Jones, 2004; Dodd \& Epstein, 2012; Epstein, 2010; Harder, 2010; Royse, 2008)

Relativamente ao ensino da investigação na formação em serviço social, podemos encontrar na literatura diferentes e variadas análise que cobrem um leque de tópicos que vão desde o estudo das metodologias para a investigação ensinadas no contexto formativo e, de que forma, podem contribuir para a investigação na prática profissional, bem como, para o interesse de estudantes e profissionais pela investigação (Knee, 2002; Lundahl, 2008; Macke \& Tapp, 2012; McCoyd et al., 2009; Phillips et al., 2012); até ao estudo da confiança sentida pelos estudantes face à investigação, ou a sua própria orientação para a investigação (Einbinder, 2014; Maschi et al., 2013; Morgenshtern et al., 2011; Stark \& Cohen, 2007).

Neste trabalho, apresentamos, essencialmente, a visão dos professores face às unidades curriculares de métodos e técnicas de investigação ao nível das abordagens metodológicas ensinadas, por se considerar estruturante compreender como se formam os estudantes, particularmente, no domínio das metodologias de investigação (qualitativa e quantitativa). Assim, entre as competências para a investigação encontra-se a necessidade de munir os estudantes, e futuros assistentes sociais, do conjunto de métodos de investigação para que estes os possam utilizar (também na sua prática), e sejam capazes de reconhecer as potencialidades/vantagens e limitações/desvantagens do uso dos métodos qualitativos, quantitativos, ou a sua combinação para, desta forma, afastar possíveis "metodologismos" ou prevalência do método sobre o objeto a investigar. Tal como refere Gambrill (1995) os métodos devem ser escolhidos de acordo com o propósito ou finalidade da investigação, pois no ensino/aprendizagem do método o foco é o conhecimento do mesmo ao invés do "marketing" que possa ser feito em torno de um ou de outro, devendo-se afastar, igualmente, possíveis confusões entre preferências pessoais e aquilo que um método oferece, pelo que a autora defende uma maior harmonização entre o propósito a investigar e o método.

A questão da metodologia ou diferentes abordagens metodológicas na formação apresenta-se como uma questão de grande relevância ao pensarmos a formação e a aplicabilidade do domínio da investigação, pois nela encerra-se a dimensão da operacionalidade na prática, mas igualmente, a articulação que esta tem com a dimensão teórica e paradigmática.

Compreender as complexidades da prática do serviço social, especialmente as suas dimensões micro e macro, só pode ser realizada pelo uso completo do repertório metodológico (Greene et al., 2009). Assim, embora possamos traçar esta visão necessária da abrangência das metodologias, estudos indicam (Fraser \& Lewis, 1993; Ryan \& Sheehan, 2000) que neste domínio, a realidade da formação pode encontrar diferentes matizes, aliás, também elas relacionadas com os contextos formativos. Por exemplo, no contexto americano observa-se a prevalência das metodologias quantitativas em serviço social, enquantoque no contexto europeu pode-se observar uma prevalência de metodologias qualitativas na investigação em serviço social. Sendo que, de uma forma global, estas últimas, parecem ter vindo a ganhar maior reconhecimento no serviço social deste o final do século XX (Padgett, 2016). 
Parte deste reconhecimento, está associado ao alinhamento que os métodos qualitativos têm com os objetivos da profissão, nomeadamente por envolverem a dimensão da escuta ativa, da observação e por mostrarem a preocupação em dar voz às pessoas em situação de vulnerabilidade (Munn, 2016), bem como, dar respostas mais adequadas à complexidade do quotidiano e das situações-problema que os assistentes sociais enfrentam.

\section{Metodologia}

Este trabalho tem como tema a investigação na formação inicial em serviço social e principal objetivo conhecer e compreender as orientações metodológicas de investigação lecionadas na formação inicial em serviço social a partir da visão dos professores. Tratase de um estudo de casos múltiplos ${ }^{1}$, exploratório ${ }^{2}$ e de natureza qualitativa ${ }^{3}$, contemplando 3 dos 17 cursos existentes de $1^{\circ}$ ciclo (licenciatura) em serviço social em Portugal. Estes representam objetos, cujos limites físicos e sociais, estão claramente determinados. Mas, também, onde o grau de enfoque se situa no momento presente, uma vez que se trata de um estudo empírico de "fenómenos" contemporâneos no seu contexto ${ }^{4}$ (Yin, 2001) e onde não se exige controle sobre eventos comportamentais (como e.g. no experimento). Efetivamente, mais do que a descrição de como se organizam os cursos estudados, procuramos explicar o porquê da cada um deles se apresentar com uma configuração específica (Bento, 2013).

$\mathrm{Na}$ orientação da nossa investigação podemos identificar duas lógicas investigação com pesos consideravelmente distintos: a lógica indutiva, que permitiu estabelecer o ponto de partida da investigação e a lógica abdutiva que nos guiou tendo em conta o objetivo do estudo.

A estratégia indutiva esteve presente, particularmente, no processo inicial de seleção dos casos. Ao procurar apurar diferenças na formação, ${ }^{5}$ intentámos identificar uma possível estrutura e mecanismo que representasse uma regularidade (Baikie, 2000) e isso foi realizado através da procura de possíveis modelos (estratégias) distintos de formação construídos a partir do desenho da estrutura dos currículos tendo em conta as UC (unidades curriculares) de investigação.

\footnotetext{
1 As exigências da confidencialidade exigiram um conjunto de cuidados que não permitem a identificação das IES.

${ }^{2}$ Yin (2001) define os estudos de caso para fins de investigação em três tipos: explicativos, descritivos e exploratórios. E, dentro destes, refere duas variações (no sentido que fazem parte da mesma estrutura metodológica do estudo de caso): os estudos de caso únicos e múltiplos, estes últimos também designados por comparativos ou, segundo Stake (2009), coletivos.

${ }^{3}$ Os estudos de caso são muitas vezes associados às metodologias qualitativas, no entanto, pode haver estudos de caso onde as metodologias quantitativas são privilegiadas (Yin, 2001). A associação ou equiparação dos estudos de caso com um tipo de método de recolha de dados específico não expressa entendimento sobre a natureza do estudo de caso enquanto estratégia de investigação (Vaus, 2001).

${ }^{4} \mathrm{O}$ contexto configura-se como particularmente fundamental para o estudo de caso (Ludwig, 2009). Embora, Yin (2001) realce a importância do contexto nos estudos de caso, atribui-lhe maior relevância em estudos de caso descritivos (pela necessidade de descrição exaustiva do fenómeno em contexto). Já Stake (2009), tendo em conta a sua divisão dos estudos de casos intrínsecos e instrumentais refere que a importância do contexto será sempre maior nos estudos de caso intrínsecos, enquanto que nos estudos de caso instrumentais a sua importância vai variar e, em alguns casos, ter pouca relevância. Importa referir que este estudo se situa no domínio dos estudos instrumentais.

${ }^{5}$ Embora a abordagem inicial pudesse ter sido construída de diferentes formas, iniciamos a aproximação através da análise documental das apresentações dos cursos nas páginas Web das IES e avaliações dos mesmos de acordo com os diversos relatórios disponibilizados pela A3ES (Agência de Avaliação e Acreditação do Ensino Superior). A primeira fonte de informação representa a possível imagem de marca e, a segunda, tem uma componente avaliativa.
} 
Procuramos, assim, analisar as regularidades entre os vários projetos de formação, e perceber como é que eles podiam ser agrupados de forma a obter/captar as regularidades/padrões entre os vários projetos de formação, o que constituiu, essencialmente, a démarche metodológica inicial.

\subsection{Estudo de Casos Múltiplos: processo de seleção dos casos}

Tendo em conta que procuramos casos diferenciados pretendíamos encontrar, no âmbito da totalidade da oferta formativa em Portugal, IES que representassem, à partida, modelos distintos de ensino de investigação em serviço social.

A determinação de quais os estudos de casos múltiplos a serem escolhidos assenta na lógica da replicação (e não na lógica da amostragem), replicação que pode ser literal ou teórica (Yin, 2001). A primeira procura/prevê resultados semelhantes (literal), já a segunda procura/prevê que sejam produzidos, por razões previsíveis, resultados contrastantes (teórica). ${ }^{6}$

O processo de seleção das IES, implicou várias fases, tendo-se partido da seguinte questão: Que critérios utilizar na seleção das IES? Isto é, que potenciais modelos distintos de formação, no domínio da investigação, nos cursos de $1^{\circ}$ Ciclo em Serviço Social se podem identificar? (lógica indutiva).

Para tal, necessitámos de definir os critérios (ou características relevantes) que nos garantissem a diversidade/heterogeneidade (Guerra, 2008) dos casos. Assim, foram adotados os seguintes critérios: i. o peso em ECTS das UC de Iniciação à Investigação no PE (Plano de Estudos); ii. o desenho curricular em Investigação das IES; iii. a dimensão histórica e consolidação do plano de estudos. ${ }^{7}$

Tabela 1. Critérios para a escolha dos casos múltiplos

\begin{tabular}{|c|c|c|}
\hline Critérios & Analisado a partir de: & Indicadores/Meios de verificação \\
\hline $\begin{array}{l}\text { Peso em ECTS das } \\
\text { UC de Iniciação à } \\
\text { investigação no PE }\end{array}$ & $\begin{array}{l}\text { PE das } 17 \text { licenciaturas } \\
\text { em Serviço Social }\end{array}$ & $\begin{array}{l}\text { № de ECTS das UC de iniciação à } \\
\text { investigação no PE. Para a sua } \\
\text { determinação recorreu-se às: } \\
\text { Fichas das Unidades Curriculares de UC com } \\
\text { designações relacionadas com a investigação e } \\
\text { outras que Ihes pudessem ser associadas. }\end{array}$ \\
\hline $\begin{array}{l}\text { Desenho curricular } \\
\text { em relação às UC de } \\
\text { Investigação das IES }\end{array}$ & $\begin{array}{l}\text { Fichas das Unidades } \\
\text { Curriculares (FUC) } \\
\text { Divisão das UC em quatro } \\
\text { grandes grupos }\end{array}$ & $\begin{array}{l}\text { 1) UC de base em Métodos e Técnicas de } \\
\text { Investigação em Ciências Sociais (MTICS); 2) } \\
\text { Seminários de Investigação; 3) Seminários de } \\
\text { Investigação e Intervenção e 4) UC Oficinais. }\end{array}$ \\
\hline Dimensão Histórica & $\begin{array}{l}\text { História do curso de } \\
\text { Licenciatura de Serviço } \\
\text { Social em cada IES }\end{array}$ & Ano de criação do curso de licenciatura. \\
\hline
\end{tabular}

${ }^{6}$ No entanto, o facto de podermos fazer esta distinção não significa que num mesmo estudo não possamos utilizar ambas.

7 Existem, em Portugal, 17 IES com o curso de 1ำ Ciclo em Serviço Social, que podem ser diferenciadas tendo em conta o tipo de ensino em que se enquadram (universitário público, privado e concordatário; politécnico público e privado) e a sua localização geográfica. Estes critérios que são, de alguma forma distintivos, não foram considerados enquanto critérios a serem utilizados, uma vez que, face aos objetivos definidos, não são estruturalmente relevantes para o estabelecimento de modelos diferentes no ensino da investigação na formação inicial em Serviço Social. Embora pudéssemos ter em conta outras características distintivas, foram estes três critérios de escolha das IES que representaram a combinação mais adequada aos objetivos traçados, tendo em conta que, tal como refere Stake (2009: 20) "é provável que as características relevantes sejam tão numerosas que apenas algumas combinações possam ser incluídas". 
Assim, o estabelecimento dos modelos de acordo com a estrutura do PE/UC de investigação centrou-se nas UC que designamos de "Base" e que incluem as UC de Métodos e Técnicas de Investigação em Ciências Sociais (MTICS) ${ }^{8}$ e Estatística/Métodos Quantitativos, em articulação com duas configurações distintas de Seminários, considerados pelas suas estruturas curriculares e conteúdos programáticos: os Seminários de Investigação (SI) e os Seminários de Investigação e Intervenção (SII), por se ter verificado, depois de uma análise mais aprofundada a partir das FUC, que as UC Oficinais estudadas referiam a dimensão da investigação de forma residual.

Desta análise, classificamos as IES em três modelos principais, a saber: i) o modelo organizado em torno de UC de Base de investigação, com 8 IES; ii) o modelo organizado em torno de UC de Base de investigação e Seminário(s) de Investigação, com 6 IES; iii) o modelo organizado em torno de UC de Base de investigação e Seminário(s) de Investigação e Intervenção, com 3 IES. Assim, em termos de pesquisa empírica foi selecionada uma IES de cada um dos "modelos" de formação (tendo em conta os três critérios referidos na tabela 1).

\subsection{Planeamento do Estudo de Casos Múltiplos: preparação e treino}

A fase de preparação para a recolha de dados, passou pela construção de um protocolo de investigação para cada uma das IES, onde as questões éticas da investigação foram abordadas, nomeadamente, a apresentação dos consentimentos informados a aplicar. Após contacto com as instituições foi decidido que a identidade das mesmas não seria revelada. Antes mesmo de iniciarmos a recolha de dados nas IES selecionadas, fizemos um estudo de caso piloto de forma a desencadear processos de treinamento da investigação (Pocinho, 2012; Yin, 2001). Para o estudo piloto selecionamos um curso de $1^{\circ}$ Ciclo em Serviço Social cuja escolha seguiu o critério da conveniência quer pela facilidade de acesso aos dados/entrevistados, quer pela proximidade geográfica da IES em causa.

No processo de desenvolvimento do estudo piloto foi possível aperfeiçoar o plano de recolha de dados, bem como, os instrumentos de recolha, pelo que esta visão integradora de todo o processo de investigação faz com que o estudo piloto, não possa ser confundido com apenas pré-testes aos instrumentos (Yin, 2001).

\subsection{Recolha de dados: fontes de evidências e participantes}

Em cada uma das unidades de análise (i.e. as três IES que fazem parte do nosso estudo), recorremos a fontes de evidências como as entrevistas e fontes documentais. Sendo que, quanto mais diversificadas forem essas fontes de evidências mais se garante uma análise mais robusta dos resultados através do uso da triangulação metodológica (Bechhofer \& Paterson, 2000), o que ajuda, igualmente, a responder à complexidade associada aos estudos de caso (Alves-Mazzotti, 2006). Neste recorte da investigação que apresentamos, constam essencialmente os dados recolhidos nas entrevistas realizadas aos docentes das UC de Investigação e coordenadores das licenciaturas. Pese embora, tenha sido também analisado um conjunto de documentação por caso, as entrevistas realizadas aos diversos professores ligados às UC de Investigação são a principal fonte de evidências desta investigação.

O número de entrevistados dependeu do número de professores associados a cada UC observando-se, por regra, que em caso de UC com a mesma designação entre o primeiro e segundo semestre (e.g. Seminário de Investigação I e II) os professores são os mesmos, reduzindo o número de professores a serem entrevistados.

\footnotetext{
${ }^{8}$ A designação genérica que atribuímos não é necessariamente igual à designação das UC em cada uma das IES.
} 
No total, no domínio das UC em análise, foram entrevistados 12 professores (Caso A: 2; Caso B: 3; Caso C: 4) e os coordenadores. Na tabela 2 apresentamos em maior detalhe as fontes de evidência segundo as unidades de análise.

Tabela 2. Fontes de Evidências segundo as Unidades de Análise

\begin{tabular}{|c|c|}
\hline Unidades de Análise & Fontes de evidências \\
\hline $\begin{array}{c}\text { Caso A } \\
\text { (UC de Base de MTICS) }\end{array}$ & $\begin{array}{l}\text { Entrevistas (3 professores): } \\
\text { - Coordenador do curso } \\
\text { - Professor de MTICS I e II } \\
\text { - Professor de Estatística/Métodos Quantitativos } \\
\text { Documentação: } \\
\text { - Fichas das UC } \\
\text { - Trabalhos de alunos disponibilizados (MTICS e Estatística). } \\
\text { - Artigo escrito por docentes da IES } \\
\text { - Documentação da A3ES }\end{array}$ \\
\hline $\begin{array}{c}\text { Caso B } \\
\text { (UC de Base de } \\
\text { MTICS \& Seminário } \\
\text { de Investigação) }\end{array}$ & $\begin{array}{l}\text { Entrevistas (4 professores): } \\
\text { - Coordenador do curso } \\
\text { - Professor de MTICS II } \\
\text { - Professor do Seminário de Investigação I e II } \\
\text { - Professor de Estatística/Métodos Quantitativos } \\
\text { Documentação: } \\
\text { - Fichas das UC } \\
\text { - Documento com os temas dos trabalhos de Seminário de } \\
\text { Investigação/Projetos de investigação desenvolvidos pelos } \\
\text { alunos na UC de Investigação em Serviço Social } \\
\text { - Documentação da A3ES }\end{array}$ \\
\hline $\begin{array}{l}\text { Caso C } \\
\text { (UC de Base de MTICS \& } \\
\text { Seminário de Investigação) }\end{array}$ & $\begin{array}{l}\text { Entrevistas (5 professores): } \\
\text { - Coordenador do curso } \\
\text { - Professores de MTICS } \\
\text { - Professor de Estatística/Métodos Quantitativos } \\
\text { - Professores dos Seminários de Investigação e Intervenção I e } \\
\text { II (são simultaneamente orientadores de estágio). } \\
\text { Documentação: } \\
\text { - Fichas das UC } \\
\text { - Trabalhos de final de curso/estágio } \\
\text { - Documentação da A3ES }\end{array}$ \\
\hline
\end{tabular}

\subsection{Recolha, tratamento e análise dos dados}

A análise qualitativa de dados surge na nossa investigação como a técnica utilizada para a compreensão e análise das entrevistas e dos documentos recolhidos, pois esta é uma técnica de tratamento da informação que permite a desconstrução do discurso e a produção de um novo discurso através de um processo de localização-atribuição de traços de significação, isto é, permite-nos tratar de uma forma metódica as informações e testemunhos (Guerra, 2008). Como software de apoio à análise qualitativa de dados utilizámos o MAXQDA cuja utilização potencia, em muito, o trabalho de investigação. Embora, tal como refere Guerra (2008), não dispensa a análise categorial e tipológica tradicional. Algumas das categorias de análise e subcategorias foram estabelecidas a priori e outras a posteriori, emergindo neste caso da análise das entrevistas aos professores e de modo a dar conta das suas racionalidades e práticas, apresentadas na tabela 2. 
Tabela 3. Categorias de Análise e Subcategorias

\begin{tabular}{ll}
\hline \multicolumn{1}{c}{ Categorias em análise } & \multicolumn{1}{c}{ Subcategorias } \\
\hline $\begin{array}{l}\text { Orientações } \\
\text { metodologias de } \\
\text { investigação lecionadas }\end{array}$ & $\begin{array}{l}\text { Metododogia qualitativa } \\
\text { Metodologia quantitativa } \\
\text { Transversalidade das metodologias ensinadas } \\
\text { Predominância de determinadas metodologias nos trabalhos dos estudantes }\end{array}$ \\
$\begin{array}{l}\text { Conceções } \\
\text { dos/as }\end{array}$ & $\begin{array}{l}\text { Perfil do/a professor/a enquanto investigador/a } \\
\text { Professores/as }\end{array}$ \\
$\begin{array}{l}\text { Visão sobre as preferências dos estudantes } \\
\text { Softwares de } \\
\text { investigação e de apoio } \\
\text { à mesma (ensinados e } \\
\text { utilizados) }\end{array}$ & $\begin{array}{l}\text { Software de investigação qualitativo } \\
\text { Gestores bibliográficos }\end{array}$ \\
\hline
\end{tabular}

No sentido de proceder à análise qualitativa de dados, optamos por fazer a transcrição integral das entrevistas que foram gravadas em áudio, uma vez que a consideramos como um importante passo para a fase da análise de dados (Alcock \& Iphofen, 2007). Esse processo de transcrição obedeceu a uma reflexão sobre o protocolo a seguir e o tipo de transcrição a efetuar.

\section{Resultados e Discussão}

\subsection{Orientações metodológicas e conceções dos professores}

Nos casos estudados, relativamente às metodologias de investigação, as IES posicionamse no campo abrangente das abordagens de investigação quantitativas e qualitativas. Neste domínio, verificou-se uma convergência entre os três casos que foi expresso de forma unânime como: os estudantes devem ter um conhecimento transversal das metodologias de investigação, isto é, conhecerem as abordagens quantitativas e qualitativas. Orientação realçada como fundamental, na medida em que, um profissional cujo perfil inclui a possibilidade de na sua prática de desenvolver investigações necessita que estas resultem da relevância das questões/objetivos que emergem da prática e não de métodos estabelecidos como relevantes a priori na formação (Greene et al., 2009; McCoyd et al., 2009).

Este posicionamento geral não significa, contudo, que as IES, não privilegiem abordagens metodológicas específicas decorrentes quer das preferências metodológicas dos professores, quer das visões destes sobre as metodologias tendencialmente mais relevantes para os assistentes sociais na relação com o contexto formativos.

Relativamente às preferências metodológicas dos professores foram identificadas, nos depoimentos, dois aspetos de convergência entre os casos estudados:

1. De uma forma geral (i.e. UC de MTICS e Seminários) a inclinação dos professores entrevistados, relativamente às metodologias de investigação, pendem para as metodologias qualitativas, tendência relacionada com as investigações que desenvolvem nas suas áreas de especialização enquanto investigadores em Ciências Sociais. O que é apontado é que, conscientes desta preferência, procuram que esta não seja indutora ou determinante na escolha das metodologias que os estudantes terão de adotar nos seus trabalhos. 
2. Apenas os professores da áreas das UC de estatística ou métodos quantitativos, se encontram voltados (pelas suas áreas de formação e como investigadores) para as abordagens quantitativas, destacando as vantagens e importância de se potenciar estas abordagens para os estudantes de Serviço Social (na investigação e intervenção), no entanto, referem que observam uma tendencial relevância atribuída às abordagens qualitativas (referindo ainda a possibilidade das abordagens mistas serem uma mais-valia a ser reforçada na prática).

Quanto à perspetiva defendida pelos professores sobre as metodologias tendencialmente mais relevantes para os assistentes sociais foram identificadas visões convergentes entre os casos $\mathrm{B}$ e $\mathrm{C}$ diferentemente do Caso $\mathrm{A}$ tendo em conta a perspetivas dos professores das UC-base de MTICS.

No Caso B, como se referiu, são "privilegiadas" (mais utilizadas) as metodologias qualitativas nos projetos desenvolvidos pelos estudantes. Esta predominância surge quer associada à sua relevância para o Serviço Social (face, até, à natureza dos dados recolhidos na sua prática) (EP2_CB), quer, em contexto formativo, pela visão errónea dos estudantes ao associarem as metodologias qualitativas a procedimentos de mais fácil utilização (EP2_CB), e que não pode ser dissociada, também, a dificuldades e resistências ao domínio da estatística/matemática, quer ainda pelos próprios objetos de estudo em Serviço Social (EP3_CB).

Neste mesmo sentido, o caso $\mathrm{C}$ destaca as metodologias qualitativas como sendo das mais utilizadas pelos estudantes, no entanto, tal como refere uma das Professoras: "Predominam [as metodologias qualitativas], embora não dominem" (EP2_CC). Este "predomínio" das metodologias qualitativas está relacionada segundo os Professores pela ligação da profissão à subjetividade dos fenómenos (EP5_CC); à prevalência do paradigma compreensivo (EP4_CC); pelo contacto privilegiado com as pessoas e 0 interesse em obter informação em profundidade. Tendo em conta estas questões uma das Professoras (EP2_CC) reconhece que, porventura, esta visão dos interesses do Serviço Social em termos de investigação sejam transmitidos explicita ou implicitamente aos estudantes. As opções metodológicas dos projetos dos estudantes, tal como referido no Caso B, algumas vezes prendem-se também com a "ilusão" dos próprios estudantes acerca da análise de dados qualitativos como sendo mais fácil de concretizar que a análise de dados quantitativos.

No caso $A$, a reflexão realizada pelos professores concentrou-se, essencialmente, no ressurgimento das metodologias quantitativas no domínio das práticas e da investigação de uma forma geral. No caso do professor de estatística (EP2 CA) cujo envolvimento na IES passa também por potenciar e apoiar projetos que a escola desenvolve junto à comunidade (apoio a planos municipais e diagnósticos concelhios) e de uma visão de conjunto das publicações internacionais e de necessidade de publicação pelos docente, para as quais o recurso a metodologias quantitativas pode ser um elemento facilitador, bem como, o acompanhamento de estudantes que utilizam metodologias quantitativa nos seus projetos e trabalhos académicos, está na base da sua visão de alteração das tendência da própria IES uma vez que, no passado, se assistia a uma mais clara prevalência das metodologias qualitativas a vários níveis.

Este professor considera, sem desvalorização das metodologias qualitativas, que as metodologias quantitativas podem ser potenciadoras da investigação na prática dos assistentes sociais, nomeadamente com recurso a bases de dados já existentes nas organizações. Na mesma linha de pensamento, situa-se o professor responsável pelas UC de MTICS (EP3_CA), que embora tenha reforçado o seu posicionamento crítico sempre que se manifestam tendências de "valorização" de determinadas metodologias por relação a outras, na medida em que, "cada uma tem as suas potencialidades e os seus limites" (Ea3). Este docente considera que atualmente se observa esse ressurgimento das metodologias quantitativas, porventura, por duas ordens de razões. A primeira prende-se com a influência da Evidence Based Practice (EBP) e a segunda está relacionada com o interesse das próprias organizações na análise das suas bases de dados, na análise dos graus de satisfação dos clientes (centrados nos modelos de gestão da qualidade). 
A coordenação do curso (caso A) aponta, ainda, a prevalência das metodologias qualitativas como mais próximas dos interesses de investigação e do agir dos profissionais, mas, também, porque as exigências de alguns tipos de investigações quantitativas, mais extensivas, segundo a entrevistada, não favorecem a sua aplicabilidade no quotidiano profissional.

\subsection{Softwares de apoio à investigação}

Os softwares para a investigação são entendidos como importantes recursos, não sendo exceção no domínio da formação em Serviço Social, uma vez que facilitam, de diversas formas, o trabalho e o tempo exigido a algumas das tarefas que um investigador terá de cumprir, o mesmo se aplicando a um interventor-investigador cujas exigências de tempo da sua prática profissional podem reforçar a necessidade de uso destas tecnologias, até para potenciar trabalhos colaborativos com outros investigadores. No entanto, o sucesso na utilização deste recurso está dependente da prévia aprendizagem dos aspetos metodológicos e técnicos subjacentes.

Os softwares para a investigação qualitativa são fundamentais para a potencialização $e$ otimização do estudo a desenvolver. Contudo, como software não dispensa a expertise do investigador que tem de dominar teórica e metodologicamente os processos. Veja-se, no caso da análise de dados qualitativos que se trata de um exercício cognitivo de pensar, de imaginar, de ter capacidade criativa. Assim, não é o software que faz a análise dos dados, mas sim o peopleware (Jennings, 2007). Todavia, esse mesmo processo, pode ser mais facilmente apreendido ou aprendido pelos estudantes com recurso a estes softwares.

Através dos depoimentos dos professores fica claro, também, o recurso a softwares de análise de dados quantitativos, que os docentes os entendem como uma ferramenta fundamental e com potencial uma vez que permite que estudantes não necessitem de dominar todo o teorema matemático subjacente a determinados cálculos para analisar os dados, o que se revela de especial interesse para estudantes das áreas das ciências sociais, pois como a literatura sublinha "as duas áreas em que os estudantes mais precisam de adquirir competências são, em primeiro lugar, como decidir quais os procedimentos estatísticos que se devem usar para responder a cada necessidade e, em segundo lugar, como interpretar os resultados obtidos" (Bryman \& Cramer, 2003, p. xxi).

Todas as IES referiram usar programas de análise de dados, onde se destacam, os de análise quantitativa, mais fortemente enraizados, comparativamente aos softwares de investigação qualitativa. Relativamente aos softwares de investigação Qualitativa a situação parece distinta, na medida em que, são menos referenciados. No caso $A$, não houve indicação de uso. No caso $B$ há indicação que ensinam e incentivam à utilização de um software de investigação qualitativa nas UC de MTICS e Seminário de Investigação II. No caso $C$, embora não utilizem, reconhecem que é importante que os estudantes comecem a utilizar estes softwares, aspeto que vem sendo refletido entre os docentes, estando prevista a realização de uma formação complementar para os estudantes.

No âmbito dos softwares de apoio à investigação, o CASO B e C referiram, ainda, a utilização de gestores bibliográficos. E, no Caso A, foram destacadas as ferramentas para a realização e envio de questionários online como importantes recursos para os trabalhos académicos e para a investigação.

\section{Conclusões}

Dos casos analisados, cuja configuração em termos de estrutura dos Planos de Estudo, deu origem à seleção dos casos a estudar, foi possível compreender que, no domínio da metodologia de investigação ensinada, não se verificaram diferenças significativas. A preocupação reportada pelos professores foi o de contribuir para o desenvolvimento de competências no domínio da investigação, visão defendida por autores que consideram importante o conhecimento de um espectro amplo das metodologias de investigação por parte dos estudantes de serviço social (Gambrill, 1995; Greene et al., 2009). 
Assim, a principal orientação encontra-se na preocupação que as IES demonstraram em garantir aos estudantes um conhecimento transversal das diferentes abordagens metodológicas, independentemente das tendências que marcam atualmente as investigações produzidas pelos assistentes sociais, o seu interesse ou adequação à área disciplinar, e, as metodologias preferencialmente adotadas pelos próprios professores enquanto investigadores.

Não obstante esta transversalidade detetada parece encontrar-se uma predominância das metodologias qualitativas, ou, a valorização das mesmas no ensino da investigação em serviço social em Portugal, pela proximidade que estas metodologias têm com os objetivos e os interesses de investigação no agir profissional, em linha com as visões de Munn (2016) e Padgett (2016).

A abordagem metodológica que adotamos nesta investigação é distinta relativamente a dois estudos de referência neste domínio, o de Fraser e Lewis (1993) nos EUA e o de Ryan e Sheehan (2000) na Austrália, ambos com recurso a metodologias quantitativas a partir da aplicação de questionários. Esses estudos procuraram, essencialmente, os tipos de investigações e metodologias ensinadas de forma a mapear o ensino da investigação em serviço social. A adoção de outra abordagem metodológica de natureza qualitativa como o estudo de casos múltiplos que apresentamos, permitiu-nos compreender, a partir dos discursos dos professores, a sua visão sobre o ensino da metodologia, as suas preferências e como associam, ou veem, a integração dos conhecimentos aprendidos pelos alunos, futuros profissionais, na sua prática. Nesta lógica, e para estudos futuros consideramos que seria útil, depois de estudados os casos apresentados (definidos através de uma lógica de replicação teórica) podermos, dentro de cada um deles, definir novos casos numa lógica de replicação literal. Podendo, contudo, ser relevante em futuros estudos adotar, também, metodologias que permitam um maior aprofundamento das nuances e particularidades da investigação na formação dos estudantes, o que poderá passar por uma abordagem mais etnográfica sobre as práticas formativas e/ou outros importantes processos formativos neste domínio auscultando não só os professores, mas também os alunos.

\section{Referências}

Adam, N., Zosky, D. L., \& Unrau, Y. A. (2004). Improving the research climate in social work curricula. Journal of Teaching in Social Work, 24(3-4), 1-18. https://doi.org/10.1300/J067v24n03_01

Alcock, J., \& Iphofen, R. (2007). Computer-assisted software transcription of qualitative interviews. Nurse Researcher, 15(1), 16-26. https://doi.org/10.7748/nr2007.10.15.1.16.c6051

Alves-Mazzotti, A. J. (2006). Usos e abusos dos estudos de caso. Cadernos de Pesquisa, 36(129), 637-651. https://doi.org/10.1590/S0100-15742006000300007

Bechhofer, F., \& Paterson, L. (2000). Principles of research design in the social sciences. Routledge.

Bento, A. v. (2013). 10 Tópicos (e dicas) sobre investigação. A. V. Bento.

Bolin, B. L., Lee, K. H., GlenMaye, L. F., \& Yoon, D. P. (2012). Impact of research orientation on attitudes toward research of social work students. Journal of Social Work Education, 48(2), 223-243. https://doi.org/10.5175/JSWE.2012.200900120

Bryman, A., \& Cramer, D. (2003). Análise de dados em ciências sociais: Introdução às técnicas utilizando o SPSS para Windows (3rd ed.). Celta Editora.

D'Cruz, H., \& Jones, M. (2004). Social work research: Ethical and political contexts. Sage.

Dodd, S.-J., \& Epstein, I. (2012). Practice-based research in social work: A guide for reluctant researchers. Routledge. 
Einbinder, S. D. (2014). Reducing research anxiety among MSW students. Journal of Teaching in Social Work, 34(1), 2-16. https://doi.org/10.1080/08841233.2013.863263

Epstein, I. (2010). Clinical data-mining: Integrating practice and research. Oxford University Press.

Fraser, M. W., \& Lewis, R. E. (1993). Research education in M.S.W. programs. Journal of Social Service Research, 17(3-4), 71-90. https://doi.org/10.1300/J079v17n03_04

Gambrill, E. (1995). Less marketing and more scholarship. Social Work Research, 19(1), 38-47. http://www.jstor.org/stable/42659917

Greene, J. C., Sommerfeld, P., \& Haight, W. L. (2009). Mixing methods in social work research. In I. Shaw, K. Briar-Lawson, J. Orme, \& R. Ruckdeschel (Eds.), The SAGE handbook of social work research. SAGE Publications.

Guerra, I. C. (2008). Pesquisa qualitativa e análise de conteúdo - Sentidos e formas de uso. Principia.

Harder, J. (2010). Overcoming MSW students' reluctance to engage in research. Journal of Teaching in Social Work, 30(2), 195-209. https://doi.org/10.1080/08841231003705404

Harvey, D., Plummer, D., Pighills, A., \& Pain, T. (2013). Practitioner research capacity: A survey of social workers in northern Queensland. Australian Social Work, 66(4), 540554. https://doi.org/10.1080/0312407X.2012.754916

Jennings, B. M. (2007). Qualitative analysis: A case of software or 'peopleware?' Research in Nursing \& Health, 30(5), 483-484. https://doi.org/10.1002/nur.20238

Knee, R. T. (2002). Can service learning enhance student understanding of social work research? Journal of Teaching in Social Work, 22(1-2), 213-225. https://doi.org/10.1300/J067v22n01_14

Ludwig, A. C. W. (2009). Fundamentos e prática de metodologia científica. Editora Vozes.

Lundahl, B. W. (2008). Teaching research methodology through active learning. Journal of Teaching in Social Work, 28(1-2), 273-288. https://doi.org/10.1080/08841230802179373

Macke, C., \& Tapp, K. (2012). Teaching research to MSW students: Effectiveness of the team-based learning pedagogy. Journal of Teaching in Social Work, 32(2), 148-160. https://doi.org/10.1080/08841233.2012.668161

Maschi, T., Wells, M., Yoder Slater, G., MacMillan, T., \& Ristow, J. (2013). Social work students' Research-related anxiety and self-efficacy: Research instructors' perceptions and teaching innovations. Social Work Education, 32(6), 800-817. https://doi.org/10.1080/02615479.2012.695343

McCoyd, J. L. M., Johnson, Y. M., Munch, S., \& LaSala, M. (2009). Quantocentric culture: ramifications for social work education. Social Work Education, 28(8), 811-827. https://doi.org/10.1080/02615470802478238

Morgenshtern, M., Freymond, N., Agyapong, S., \& Greeson, C. (2011). Graduate social work students' attitudes toward research: Problems and prospects. Journal of Teaching in Social Work, 31(5), 552-568. https://doi.org/10.1080/08841233.2011.615287

Munn, J. C. (2016). Teaching qualitative methods to social workers: Four approaches. Qualitative Social Work, 15(3), 322-330. https://doi.org/10.1177/1473325015617008

Padgett, D. K. (2016). Qualitative methods in social work research (3rd ed.). Sage.

Phillips, J., MacGiollaRi, D., \& Callaghan, S. (2012). Encouraging research in social work: narrative as the thread integrating education and research in social work. Social Work Education, 31(6), 785-793. https://doi.org/10.1080/02615479.2012.695200 
Vol. 7 | Investigação Qualitativa em Educação: Avanços e Desafios

Pocinho, M. (2012). Metodologia de investigação e comunicação do conhecimento científico. Lidel.

Royse, D. (2008). Research methods in social work (5th ed.). Thomson Brooks/Cole.

Ryan, M., \& Sheehan, R. (2000). Research education in australian BSW programs: Results of a survey. Advances in Social Work, 3(1), 136-151.

Stake, R. E. (2009). A Arte da investigação com estudos de caso (2nd ed.). Fundação Calouste Gulbenkian.

Stark, R. B., \& Cohen, B. E. (2007). Promoting positive student attitudes toward social Work research using course web sites. Journal of Teaching in Social Work, 27(1-2), 181198. https://doi.org/10.1300/J067v27n01_12

Vaus, D. A. de. (2001). Research design in social research. SAGE.

Yin, R. K. (2001). Estudo de caso: Planejamento e métodos (2nd ed.). Bookman. 